\title{
Tetraethyl Orthosilicate and Acrylic Acid Forming Robust Carboxylic Functionalitities on Plastic Surfaces for Biodiagnostics ${ }^{a}$
}

Conor Coyle, Ram Prasad Gandhiraman*, Vladimir Gubala, Nam Cao Hoai Le, Christy Charlton O'Mahony, Colin Doyle, Bryony James, Paul Swift, Stephen Daniels, David E. Williams

C. Coyle, R.P. Gandhiraman, V. Gubala, N.C. H. Le, C. Charlton O’Mahony

Biomedical Diagnostics Institute (BDI), Dublin City University, Dublin 9, Republic of Ireland Fax: (+353-1-700-5384); E-mail: RamPrasad.Gandhiraman@dcu.ie)

C. Doyle, B. James

Research Centre for Surface and Materials Science, Department of Chemical and Materials Engineering, University of Auckland, Auckland 1142, New Zealand

P. Swift, S. Daniels

National Centre for Plasma Science and Technology (NCPST), Dublin City University, Dublin 9, Republic of Ireland

D.E. Williams

MacDiarmid Institute for Advanced Materials and Nanotechnology, Department of Chemistry, University of Auckland, Auckland-1142, New Zealand

Surface functionalisation and effects related to non specific binding of the detection molecules are the key aspects to be considered for fluorescence-linked bioassays. Here we present a deposition of polymeric structures with carboxylic acid functionalities by plasma enhanced chemical vapour deposition.. We report on characterisation and some unique properties of film that is formed as a result of sequential, plasma assisted fragmentation and deposition of vapours of acrylic acid and tetraethyl ortho silicate (TEOS). In this context, TEOS serves as an adhesion layer to the plastic substrate and also as a network-building layer for further cross-linking with acrylic acid. The presence of silanols ( $\mathrm{Si}-\mathrm{OH})$ can facilitate large uptake of water molecules and cause significant hydration of the layer, which in combination with high total negative charge lowers the non specific binding of biomolecules.. 
The reactivity of the carboxyl functionalities was confirmed by total internal reflection ellipsometry technique in a reaction with amino terminated ssDNA. Ageing studies of the film showed long term stability over 50 days. Overall, the specific combination of TEOS and acrylic acid deposited by plasma assisted vapour method results in film with an increased percentage of carboxyl groups to $16.4 \%$ as compared to that of $6.5 \%$ in acrylic acid as determined in the core level C 1s XPS spectrum and a significant increase in signal to noise ratio.

\section{Introduction}

Considerable effort has been invested in a production of new class of thermoplastic polymer, cyclo olefin polymer (COP), for point-of-care diagnostic applications (Ma et al., 2009; Raj et al., 2009). Such polymers are of great interest because of their favourable properties, such as high glass transition temperature, low autofluoescence, optical clarity, resistance to organic solvents, low water uptake and easy machinability (Becker and Gatner, 2008; Diaz-Quijada et al., 2007). Zeonor ${ }^{\circledR}$ is a type of COP that includes these desirable properties whilst providing a cost effective platform for carrying out bioassays in disposable biodiagnostic chips. The bioassays depend strongly on antibody - antigen recognition, for which the surface-bound antibody must be efficiently immobilised in their active form. COPs consist almost exclusively of hydrocarbons and thus possess no native groups amenable for specific reaction with the capture elements. Therefore, in order to enable covalent attachment of biomolecules, such as antibodies and DNA, the COP surface needs to be functionalised. A number of methods have been described for this (Gandhiraman et al., 2010a; Volcke et al., 2010). In all cases, the specific aim was to prepare an ideal surface material that should have a high binding capacity but also show good adhesion, stability and resistance against many washing steps and regeneration conditions. Another critical characteristic such surface should possess, related to sensitivity in fluorescence based detection devices, is the ability to tune the non- 
specific binding of the non-analyte components of the sample. The net expected outcome is a decrease in the background response and effective increase of the signal to noise ratio (Gubala et al., 2010; Gandihraman et al, 2010b).

In this communication, we describe a one step process for modification of COP surface, by plasma enhanced chemical vapour deposition (PECVD). This technique has a number of advantages over the multistep, wet chemical methods or chemical vapour deposition (CVD): i) it can be used to coat large number of substrates; ii) it avoids direct contact with the solvent thus reducing chemical waste; iii) it operates at room temperature maintaining required functionality. Significant fragmentation of the feed generally occurs in glow discharges; as a result a wide range of functional groups appear in the coating. Plasma deposition and treatment processes have been previously optimised for the improvement of the adhesion, spreading and proliferation of cells at the surface of materials of biomedical interest, as well as for the improvements in membrane properties such as hydrophobic/philic character, non fouling properties, transport and capability of immobilising molecules (Favia et al., 1999; Palumbo et al., 1999). Fragmentation of the precursors can be precisely controlled by carefully tuning parameters such as power input and duty cycle to obtain a high degree of monomer structure retention in the film.

In the work presented here, surfaces of COP substrates were modified with carboxylic groups for application in biosensors. We report on a technique for the modification of substrates using tetraethyl orthosilicate (TEOS) and acrylic acid as monomer sources to produce polymer-like coatings. A comparison with the film prepared by the deposition of acrylic acid alone is presented. Polymerisation of acrylic acid to form films on various substrates has been extensively studied (Detomaso et al, 2005a; Alexander and Duc, 1998; Gupta et al., 2001). While the adhesion of the poly-acrylic acid is usually good, the polymers tend to suffer from effects of high non-specific binding. We have demonstrated in our earlier work that the combination of precursors with two distinct function was effective approach to increase the 
film stability and reactivity and consequently to gain better control over non specific binding (Gandihraman et al, 2010b). Hence the use of TEOS as an adhesion and network building layer was attempted with a sequential deposition of acrylic acid. The objective of this work was to prepare stable film with high quantity of $-\mathrm{COOH}$ groups available for reaction with biomolecules, while retaining good adhesion and low unspecific adsorption of the detection molecules. Carboxylic acid-rich polymeric structures are preferred. Upon activation with dehydrating agents, such as 1-Ethyl-3-[3-dimethylaminopropyl] carbodiimide Hydrochloride (EDC), the $-\mathrm{COOH}$ group can be used to perform an aminolysis reaction with free amines. Such groups are abundant in proteins and can be easily incorporated into the $5^{\prime}$ or $3^{\prime}$ end of oligonucleotides. The low pKa values of carboxylic acid $(\mathrm{pKa}=4.0-4.2)$ means that under standard physiological conditions, the equilibrium is shifted towards its deprotonated species, hence providing significant total negative charge on the surface. Charged films with low zeta potential can take advantage of electrostatic repulsions between the surface groups and other intrinsically negatively charged molecules, such as DNA and low isoelectric point proteins, thus effectively reducing the non-specific binding. Indeed, one of the most effective blocking agent used in immunoassays to date is bovine serum albumin (BSA) with isoelectric point in the range of $4.9-5.5$.

The surface modification procedure was characterised by contact angle measurement to monitor changes in wettability and ageing effects, fluorescence microscopy to study the nonspecific binding, XPS and FT-IR to analyse the chemical composition and the nature of chemical bonding present in the film, AFM for nanoscale topography and TIRE to investigate the thickness of the coatings and for studying biomolecule immobilisation.

\section{Experimental Part}

\section{Reagents}


COP slides (Zeonor® 1060R) 75 mm x 25 mm were supplied by Amic AB (Uppsala, Sweden). Acrylic Acid $\left(\mathrm{C}_{3} \mathrm{H}_{4} \mathrm{O}_{2}\right)$ of $99 \%$ purity and tetraethyl orthosilicate $\left(\mathrm{C}_{8} \mathrm{H}_{20} \mathrm{O}_{4} \mathrm{Si}\right)$ of 99.999\% purity were purchased from Sigma-Aldrich. Lissamine ${ }^{\mathrm{TM}}$ rhodamine B sulfonylchloride $\left(\lambda_{\mathrm{exc}}=532 \mathrm{~nm}\right.$ and $\left.\lambda_{\mathrm{em}}=550 \mathrm{~nm}\right)$ was obtained from Invitrogen. All chemicals were used as received without further purification. Gold-coated glass slides ( $\mathrm{Ti} / \mathrm{Au}$ $=2 \mathrm{~nm} / 48 \mathrm{~nm}, 26 \mathrm{~mm} \times 76 \mathrm{~mm}, 1 \mathrm{~mm}$ thick) were purchased from Phasis Sarl (Geneva, Switzerland). N-(3-dimethylaminopropyl)-N'-ethylcarbondiimide hydrochloride (EDC), NHydroxysuccinimide (NHS), and xylene were purchased from Sigma Aldrich and used without further treatment. Amino modified single stranded DNA (19 bp) was purchased from Eurofins MWG Operon (Ebersberg, Germany).

\section{Instrumentation}

\section{Plasma Enhanced Chemical Vapour Deposition (PECVD)}

Coatings were carried out in a computer controlled PECVD reactor Europlasma, model CD300 (Oudenaarde, Ghent, Belgium). An aluminium vacuum chamber was connected to a Dressler CESAR 136 RF power source with operating frequency $13.56 \mathrm{MHz}$ with automated impedance matching. Further details of the deposition system are provided elsewhere (Gandihraman et al., 2009c).

\section{Water Contact Angle (WCA)}

Contact angles were measure with a contact angle goniometer (First Ten Angstroms FTA200) using a high purity HPLC grade water (Sigma Aldrich) as the probe liquid.

\section{Atomic Force Microscope (AFM)}

Roughness measurements on COP surfaces were performed with a Digital Instruments, Veeco Metrology Group, Nanoscope Dimension ${ }^{\mathrm{TM}} 3100$ AFM in tapping mode at ambient 
conditions, using an aluminium cantilever (BudgetSensors, Bulgaria) with a resonant frequency of $300 \mathrm{kHz}$ and a spring constant of $40 \mathrm{~N} / \mathrm{m}$. For each sample, an area of $30 \times 30$ $\mu \mathrm{m}^{2}$ was imaged at a rate of $1 \mathrm{~Hz}$.

For alternative AFM data:

Surface morphology and roughness on plain COP, TEOS-coated COP, AA-coated COP and TEOS/AA-coated COP surfaces were measured with a Digital Instruments (DI) BioScope ${ }^{\mathrm{TM}}$ II (Veeco Instruments Inc., Plainview, NY, USA) in tapping mode in air. Silicon cantilevers with integrated tips (TESP, Veeco Probes, Camarillo, CA, USA) and with resonant frequencies between 327 and $349 \mathrm{kHz}$, and with $\approx 30 \mathrm{~N} / \mathrm{m}$ spring constant were used. For each surface, three locations with surface area of $2 \times 2 \mu \mathrm{m}^{2}$ each were imaged at a rate of 0.5 $\mathrm{Hz}$ and at a resolution of $512 \times 512$. Research NanoScope 7.30 software (Veeco Instruments Inc., Plainview, NY, USA) was used to analyse the measured data and estimate the RMS roughness.

\section{X-ray Photoelectron Spectroscopy (XPS)}

The XPS data were collected on a Kratos Axis UltraDLD equipped with a hemispherical electron energy analyser. Spectra were excited using monochromatic Al Ka X-rays (1486.69 $\mathrm{eV}$ ) with the X-ray source operating at $100 \mathrm{~W}$. This instrument illuminates a large area on the surface and then using hybrid magnetic and electrostatic lenses collects photoelectrons from a desired location on the surface. In this case, the analysis area was a 220 by $220 \mu \mathrm{m}$ spot. The measurements were carried out in a normal emission geometry. A charge neutralisation system was used to alleviate sample charge build up, resulting in a shift of approximately 3 eV to lower binding energy. Survey scans were collected with $160 \mathrm{eV}$ pass energy, whilst core level scans were collected with a pass energy of $20 \mathrm{eV}$. The analysis chamber was at pressures in the $10^{-9}$ torr range throughout the data collection. 
Data analysis was performed using CasaXPS (www.casaXPS.com). Shirley backgrounds were used in the peak fitting. Quantification of survey scans utilised relative sensitivity factors supplied with the instrument. Core level data were fitted using Gaussian-Lorentzian peaks (30\% Lorentzian). The binding energy scale was corrected for the neutraliser shift by using the $\mathrm{C} 1 \mathrm{~s}$ signal from saturated hydrocarbon at $285.0 \mathrm{eV}$ as an internal standard. The elements present in the coating $\mathrm{C}, \mathrm{O}$ and $\mathrm{Si}$ were detected using the XPS survey scan. High resolution scans of individual core levels showed the various bonding states.

\section{Attenuated Total Reflection Fourier Transform Infrared Spectroscopy (ATR-FTIR)}

ATR-FTIR spectra were recorded on a Perking Elmer - Spectrum GX FTIR used in the attenuated total reflection mode (Horizontal accessory equipped with a ZnSe crystal, Perkin Elmer) The detector and sample chamber were purged with nitrogen gas during measurements. For all data presented, unmodified COP slides were used as background. Scans were collected between 1600 to $400 \mathrm{~cm}^{-1}$ range with 32 scan average per image.

\section{Fluorescence spectroscopy}

Fluorescence spectroscopy was carried out using a Perkin Elmer LS 45 fluorescence spectrometer. Fluorescent images were analysed using the ScanArray Gx software (Perkin Elmer) Carboxy coated COP slides were scanned at $635 \mathrm{~nm}$ (Cy5) wavelengths using a laser-activated confocal scanner (ScanArray Gx; Perkin Elmer) at a $20 \mu \mathrm{m}$ resolution.

\section{Total Internal Reflection Ellipsometry (TIRE)}

Total internal reflection ellipsometry (TIRE) or surface plasmon enhanced ellipsometry (SPEE) is a spectroscopic ellipsometry measurement method operated under total internal reflection (TIR) condition (Nam et al., 2010) Similar to conventional spectroscopic method, TIRE also measure two ellipsometric angles $\Psi$ and $\Delta$ versus wavelengths. These $\Psi$ and $\Delta$ 
values are defined by the ratio $\rho$ of the reflection coefficients $R_{p}$ and $R_{s}$ for components of light polarised parallel $-p$ and perpendicular $-s$ to the plane of incidence following the ellipsometry equation (Azzam and Bashara, 1992)

$\rho=\frac{R_{p}}{R_{S}}=\tan \Psi \exp (i \Delta)$

Where $R_{p}$ and $R_{s}$ are the electric field reflection coefficients for $-p$ and $-s$ polarised lights. A COP slide was cut into small pieces and dissolved in xylene at $0.25 \mathrm{wt} \% \mathrm{w} / \mathrm{v}$ to make the COP solution. The COP solution was then spin coated onto the Au-coated glass slide at 1300 rpm in $30 \mathrm{~s}$ with acceleration in $2 \mathrm{~s}$ and 2000 in $5 \mathrm{~s}$ with acceleration in $2 \mathrm{~s}$ (Larsson et al., 2007). The solvent xylene was naturally evaporated, leaving a thin COP layer of approximately $23 \mathrm{~nm}$ in thickness measured by a spectroscopic ellipsometer (UVISEL, JobinYvon Horiba, France). The substrate was subsequently introduced to an oxygen plasma treatment for 1 minute, during which its thickness was reduced to $10 \mathrm{~nm}$. TEOS and acrylic acid was then deposited onto the substrate in the same PECVD chamber with the same conditions as for the COP slides. The substrate was then assembled into a specialised flowcell setup with a BK7 prism for TIRE measurements with the UVISEL spectroscopic ellipsometer. The flow-cell contains three wells on the same TEOS and acrylic acid functionalised COP surface for three TIRE measurements at angle of incidence of $70^{\circ}$ with wavelengths ranging from 500 to $900 \mathrm{~nm}$. First, we performed stability test of TEOS and acrylic acid surface under continuous immersion in PBS buffer (pH 7.0) in well 1. $\Psi$ and $\Delta$ spectra were measured after PBS buffer was pumped in and replaced in well 1 after $1 \mathrm{hr}$ and $2 \mathrm{hr}$. The baseline $\Psi$ and $\Delta$ spectra were also measured for TEOS and acrylic acid surface in well 2 and 3 filled with PBS buffer before DNA attachment. $30 \mu$ ls of aminated 19 bpssDNA $10 \times 10^{-6} \mathrm{M}$ in MES buffer ( $\mathrm{pH} 8.0$ ) without EDC and with $50 \mathrm{mM}$ EDC were then pumped in well 2 and 3, respectively, and allowed to react with TEOS and acrylic acid surface for $1 \mathrm{hr} . \Psi$ 
and $\Delta$ spectra were recorded for well 2 and 3 after $1 \mathrm{hr}$ of reaction. Final $\Psi$ and $\Delta$ spectra were recorded for well 2 and 3 before they were rinsed extensively with PBS.

\section{Total internal reflection fluorescence (TIRF) microscopy}

A $473 \mathrm{~nm}$ blue laser with a maximum output of $50 \mathrm{~mW}$ (Photop Technologies Inc, Fujian, China) was coupled to a microscopy slide (COP or glass) through a BK7 prism (BRP-5, Newport, Oxfordshire, UK) which was index matched to the slide using immersion oil. Light entered the slide at an angle of $15^{\circ}$ from horizontal giving an angle of reflection at the slide/water interface of approximately $65^{\circ}$ from the vertical. This angle is below the critical angle of $61^{\circ}$ causing the light to undergo total internal reflection. This setup was placed above the objective on an Olympus IX81 (Olympus, Essex, UK) inverted fluorescence microscope. A drop of fluorescent dye-doped latex particle (Kisker Biotech, Steinfurt, Germany) solution was placed on top of the slide at a location where the laser beam was being reflected from the top surface giving an evanescent field at that point. As particles interact with the surface, they enter the evanescent field and are excited emitting fluorescence which is collected through the slide from below. The objective used was a 20x 0.50 NA (UPlanFLN, Olympus, Essex, UK) giving a viewing area of $0.144 \mathrm{~mm}^{2}$. An Olympus U-MWIBA filter blocked the laser excitation light and allowed collection of fluorescence emitted by the particles. Images were recorded every 10 seconds for 2000 seconds with a 5 second exposure time on an Olympus DP71 camera. Image colour was adjusted using ImageJ software to remove background noise before particles were counted using Matlab image processing software.

\section{Results and Discussion}

Two different types of coatings have been deposited in this study, (a) an acrylic acid coating using acrylic acid precursor in an argon plasma for 30 seconds at 14 watts RF power, 
hereafter called AA coating and (b) a sequential deposition of TEOS and Acrylic acid coating for 30 seconds each in argon plasma at 14 watts RF power, hereafter called TEOS \& AA. A plasma pre-treatment of 3 minutes using argon and oxygen plasma at $250 \mathrm{~W}$ was used for both cases.

Surface chemical characterisation

The nature of chemical bonding present in the film was determined by Fourier-transform infrared spectroscopy (FTIR) used in the attenuated total reflection mode (ATR). The presence of the $\mathrm{COOH}$ functionality was confirmed by ATR-FTIR (Fig.1). The TEOS coating on its own did not show any IR absorption in the carbonyl region.

Table 1. Elemental analysis by XPS

\begin{tabular}{|cccccc|}
\hline Sample & C-C & C-C & $\begin{array}{c}\text { Surface } \\
\text { Oxide }\end{array}$ & C-OH & C=O \\
\hline \multicolumn{5}{c|}{ Binding energy (eV) } \\
(\% Area of Peak) \\
\hline COP & 285.0 & 285.9 & 287.5 & - & - \\
& $(84 \%)$ & $(13.9 \%)$ & $(2.1 \%)$ & - & - \\
Acrylic & 285.0 & & & 286.5 & 289.2 \\
Acid & $(68.1 \%)$ & & & $(25.3 \%)$ & $(6.5 \%)$ \\
TEOS & 285.0 & & 286.3 & 289.3 \\
\& AA & $(60.5 \%)$ & & $(23.1 \%)$ & $(16.4 \%)$ \\
\hline
\end{tabular}

XPS survey scans confirmed the introduction of Si into the surface layer as a consequence of the TEOS deposition, and $\mathrm{O}$ as a consequence of acrylic acid deposition. A detailed study of the $\mathrm{C} 1 \mathrm{~s}$ region revealed subtle, significant differences in the chemical composition of the different layers. The elements present in the coating $(\mathrm{C}, \mathrm{O}$ and $\mathrm{Si})$ were identified by XPS survey spectra listed quantitatively in Table 1.High-resolution spectra were recorded from individual peaks. Qualitatively, the C 1s spectra for both the films (Fig 2) look reasonably similar with one saturated hydrocarbon peak $(285.0 \mathrm{eV})$ and two additional peaks of higher 
binding energy at $286.3 \mathrm{eV}, 286.5 \mathrm{eV}(\sim+1.4)$ and $289.2 \mathrm{eV}, 289.3 \mathrm{eV}(\sim+4.2)$. The +1.4 peak could be assigned to $\mathrm{C}-\mathrm{O}-\mathrm{C}$ as well as $\mathrm{C}-\mathrm{OH}$, whilst the higher binding energy peak $(\sim+4.2)$ is characteristic for $\mathrm{O}-\mathrm{C}=\mathrm{O}$ (Detomaso et al., 2005b). It is observed that the percentage area of carbonyl peaks in $\mathrm{C} 1 \mathrm{~s}$ environment is higher in TEOS \& AA (16.4\%) than to that of AA $(6.5 \%)$ for the same acrylic acid exposure time in the plasma. This demonstrates that the plasma polymerisation of acrylic acid depends strongly on the nature of the interfacial layer and the presence of a plasma polymerised siloxane intermediate layer enhances the carboxylic functionality in the coating.

\section{Surface morphology}

Film morphology is one of the factors that cause non specific binding of biomolecule, provided the surface roughness is comparable to or larger than the size of the biomolecule to be immobilised (Dudek et al., 2009; Beaulieu et al. 2009; Morra et al., 1991). A higher surface roughness implies an increased surface area that could increase the physisorption of the biomolecule as the molecule could stretch out on the rough surface. Poksinski and Advicula reported that films with roughness ranging from micrometer to nanoscale $(>5 \mathrm{~nm})$ enhance adsorption of proteins (Poksinski et al., 2007; Advicula et al., 2005). For the immobilisation of DNA and also for the ultrasensitive detection of DNA, a surface with a roughness of $5 \AA \AA$ has been used (Rechendorff et al., 2006; Wälivaara et al., 1994). The observed initial surface roughness of $1.1798 \pm 0.1151 \mathrm{~nm}$ and $1.0155 \pm 0.0724 \mathrm{~nm}$ for AA and TEOS \& AA films respectively, (Fig. 3 ) is comparable with that found for films formed from liquid phase silanisation using organosilanes on silica, mica and glass surfaces (Wen et al., 2007; Rechendorff et al., 2006; Liu et al., 2009).

\section{Surface wettability}

Plasma exposure is an effective procedure to alter surface properties for a number of materials including glass, metals and polymers while leaving the bulk properties of the material intact. 
It is known that exposing COP to a plasma regime changes it from its native hydrophobic state $\left(>90^{\circ}\right)$ to a hydrophilic state $\left(<10^{\circ}\right)$ These contact angle changes are unstable over long periods of time and will eventually return to their original state. The time scale of this process depends on the discharge power exposed to substrates (Roy et al., 2006; Hu et al., 1996). Hydrophobic recovery is a well-known phenomenon of plasma exposed polymers, which can be attributed to the rearrangement of polymer chains at the surface along with migration of low molecular weight residues (Pasternack et al., 2008). Fig.4 shows that coatings of AA and AA \& TEOS retain their hydrophilic nature for longer periods of time compared to the uncoated COP. AA retains lower water contact angle when compared to AA $\&$ TEOS.

Biomolecule immobilisation and non specific binding

The ability of the coating to covalently bind biomolecules was assessed by attaching fluorescently labelled single strand of DNA. Specific surface binding / capture efficiency was assessed through immobilisation of DNA with $\mathrm{NH}_{2}$ - group via aminolysis. Non-specific binding was determined via physical adsorption of dye labelled DNA without the $\mathrm{NH}_{2}$ group. The resistance of the coatings to washing was also investigated. A routine manipulation with substrates in immunoassays involves extensive washing with aqueous solutions, often containing detergents. Good adhesion and stability of coatings against washing and hydrolysis are necessary to ensure reproducibility and precision of a biodevice. The substrates were spotted with Cy5 labelled amino terminated DNA. The coated substrates were subjected to extensive washing with PBS Tween and deionised water, dried with nitrogen and the fluorescence intensity was measured. This process was repeated five times. Both AA and AA \& TEOS films showed reasonable adhesion strength and resistance against washing with water and PBS Tween. The signal-to-noise ratio, measured as fluorescence signal of specifically bound DNA to unspecifically adsorbed DNA, was higher for TEOS \& AA than 
that of AA (Fig $5 \mathbf{a , ~ b ) . ~ A l s o , ~ c o n s i d e r i n g ~ t h e ~ f l u o r e s c e n c e ~ i n t e n s i t i e s , ~ h i g h e r ~ d e n s i t y ~ o f ~}$ captured DNA molecules was observed on AA \& TEOS coatings. This polymer consisting of $\mathrm{COOH}$-siloxane network also showed very good adhesion to the COP surface even after execution of the washing steps.

A label-free, ellipsometric technique operated under Total Internal Reflection (TIR) condition, i.e. Total Internal Reflection Ellipsometry (TIRE), was used to corroborate the fluorescence results on the TEOS \& AA surface (Kasry et al., 2009). TIRE was also used to test the stability of TEOS \& AA surface under long exposure to PBS buffer. As shown in Fig. 6a, the $\Delta$ spectra remained the same over a period of $2 \mathrm{hrs}$ suggesting that the TEOS \& AA is indeed stable against hydrolysis in aqueous environment. Replacing the PBS buffer with solution of ssDNA resulted in a large shift in $\Delta$ spectra. (Fig. 6b). However, extensive rinsing with PBS revealed that the DNA is effectively immobilised only when the activating agent, EDC, was present in the reaction mixture. In the absence of EDC, the shift in $\Delta$ spectrum has been reduced substantially, close to the original $\Delta$ spectrum of TEOS \& AA surface. It is possible that some small amount ssDNA have been non-specifically adsorbed to the TEOS \& AA surface even without EDC activation. Fitting of $\Psi$ and $\Delta$ spectra gave corresponding thickness of ssDNA bound to the surface. It was found that with the EDC activation, the thickness of ssDNA bound to surface was $17.8 \pm 2.2(\mathrm{~A})$. while that of without EDC activation was as small as $2.6 \pm 1.4(\mathrm{~A})$.

Dye-doped nanoparticles (NP) are often used as labels in bioassays to increase signals and improve sensitivity. The benefits of sensitised NPs as bright detection molecules can only be realized if the particles have good colloidal stability and the non-specific interactions between the particles and the substrate surface is kept at minimum. The latter was of great interest in the context of the presented work. Although the commercial nanoparticles are typically negatively charged to avoid aggregation, they often show high levels of particle-surface 
binding even in absence of biomolecules (Charlton et al., 2011). We have investigated the non specific adsorption of dye-doped NPs on the AA \& TEOS surface and compared it with a standard, commercially available, epoxy-coated glass slide, commonly used in bioassays. As expected, the electrostatic forces of the negatively charged NP surface and the negatively charged $-\mathrm{COO}^{-}$groups on the substrate dominate in this binding event. Figure 8 illustrates low particle count on the AA \& TEOS surface compared with that of the epoxy slide indicating the suitability of this surface for bioassays based on fluorescent particles. It is also understandable that the unspecific adsorption effect can be modulated by changing the $\mathrm{pH}$ of the solution.

The picture that emerges from the different characterisations is that the role of the siloxane network, formed by fragmentation of TEOS that condense and polymerise on the COP surface is three-fold. First, it serves as a bonding layer to plastic substrates for enhanced adhesion. Second, it provides a reactive ground for cross-linking with acrylic acid, thus increasing the content of $-\mathrm{COOH}$ functionality. And third, the presence of silanols $(\mathrm{Si}-\mathrm{OH})$ can facilitate large uptake of water molecules and cause significant hydration of the layer, which is in combination with high total negative charge crutial parameters for low non-specific adsorption of molecules.

\section{Conclusion}

Coatings with carboxylic acid groups have been successfully deposited by plasma enhanced chemical vapour deposition on cyclo olefin polymer substrates. The sequential deposition of TEOS and AA resulted in a formation of carboxylic acid-rich surfaces providing increased reactivity of the functional groups. The binding properties have been studied using total internal reflection ellipsometry and fluorescence microscopic techniques using amino terminated ssDNA. The ageing study showed that the $-\mathrm{COOH}$ layer retained its hydrophilicity for a tested period of 50 days. The Si-O bond in TEOS served as an adhesion domain to form 
a strong covalent linkage with the hydrocarbon network of COP polymer and also as a network building layer for further functionalisation of acrylic acid. The quantitative analysis by XPS revealed that the amount of carboxylic acids available on the surface, was 2.5 higher in films containing TEOS as an intermediate layer. The advantage and applicability of such surface to immunoassays is currently being investigated and the results will be presented in due course.

Acknowledgements:

This material is based upon works supported by the Science Foundation Ireland under Grant No. 05/CE3/B754. D.E.W. is an E.T.S. Walton visiting fellow of Science Foundation Ireland.

Received: ((will be filled in by the editorial staff)); Revised: ((will be filled in by the editorial staff)); Published online: ((will be filled in by the editorial staff));

DOI: ((will be filled in by the editorial staff)) 


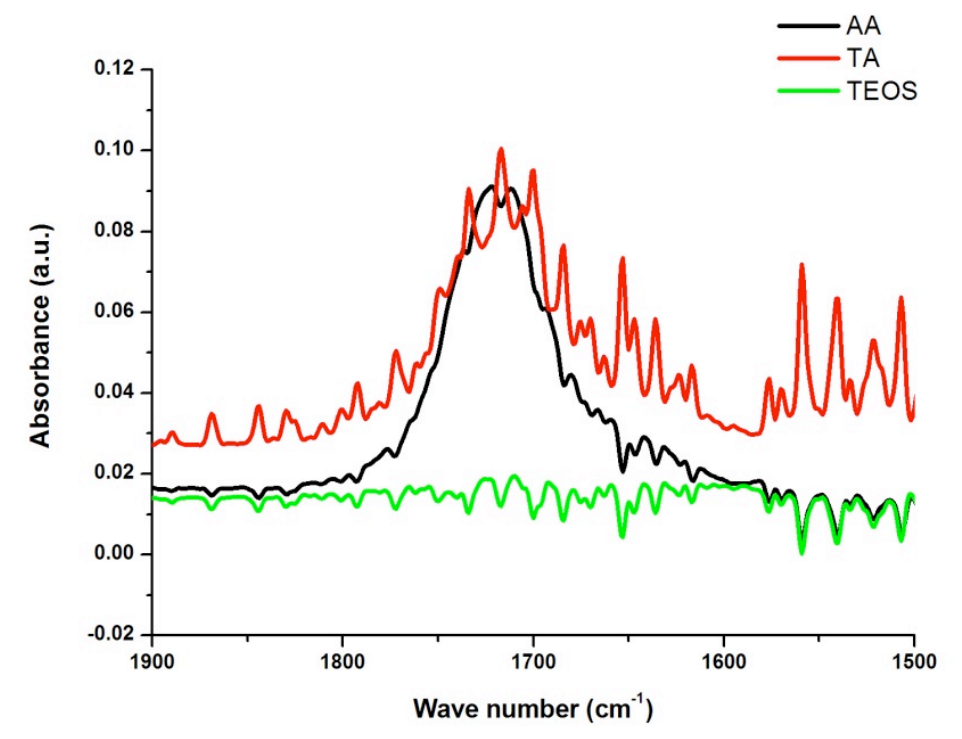

Figure 1. ATR-FTIR spectra of Acrylic acid, TEOS\&Acrylic acid and TEOS coatings on COP substrates.
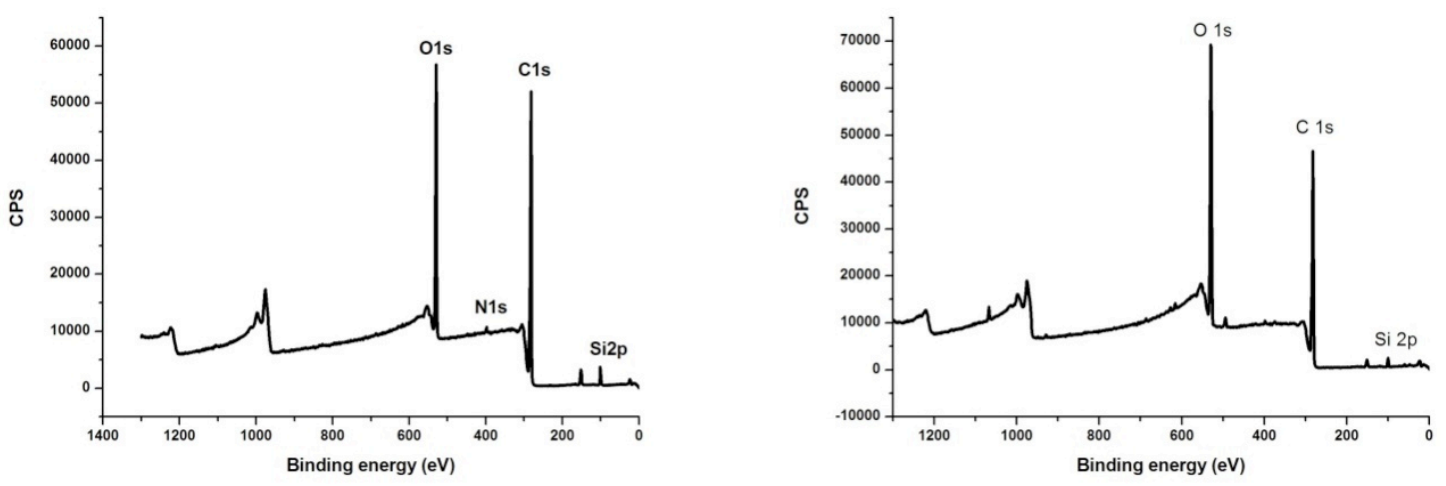

Figure 2. XPS survey scan collected with $160 \mathrm{eV}$ pass energy using monochromatic Al Ka monochromatic X-rays (left): AA and (right) TA on COP. 

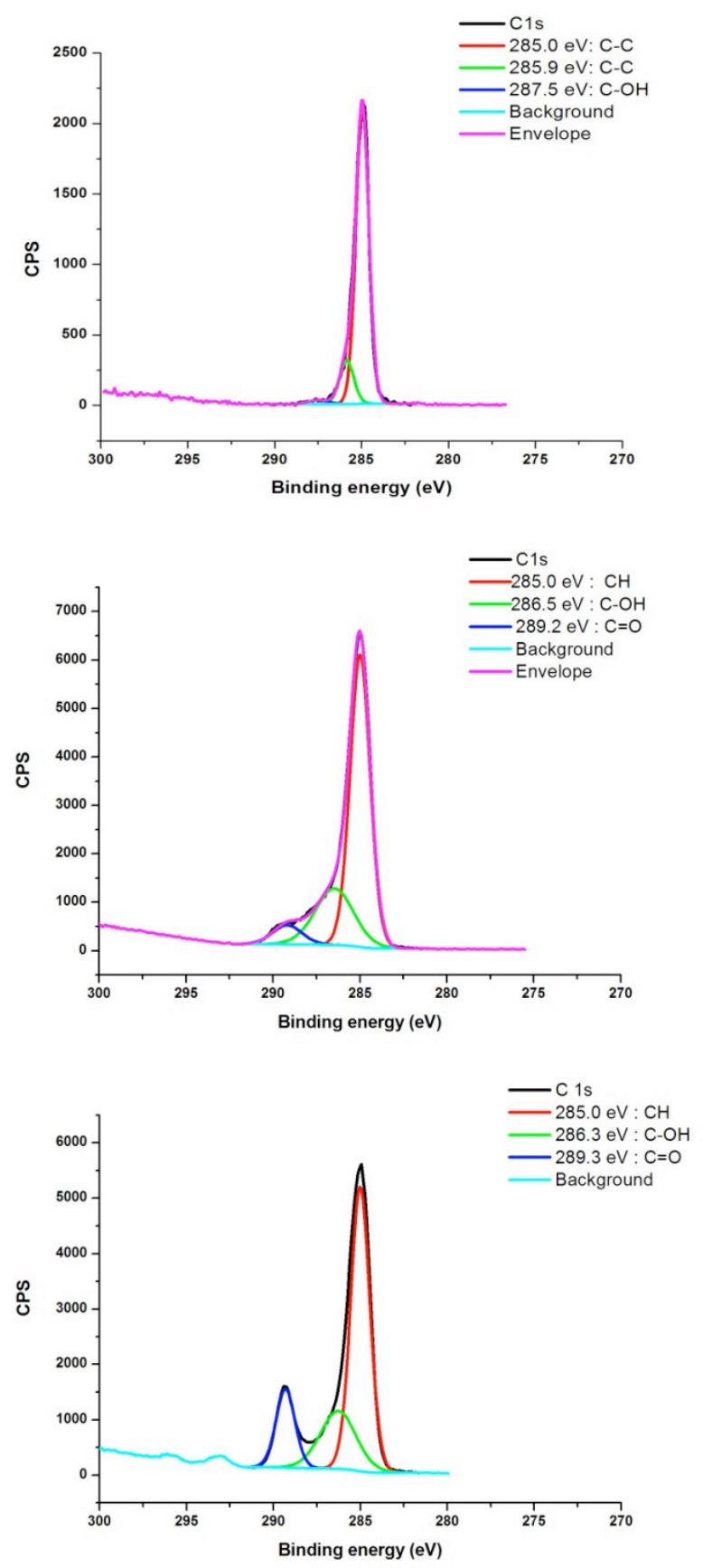

Figure 3 High resolution C 1s core level photoemission spectra of (Top) untreated COP substrate, (Middle) Acrylic acid coated COP substrate and (Bottom) TEOS \& AA coated COP substrate, taken with a pass energy of $20 \mathrm{eV}$ using monochromatic $\mathrm{Al} \mathrm{K} \alpha$ monochromatic $\mathrm{X}$ rays. The $\mathrm{C} 1 \mathrm{~s}$ peak is deconvoluted to show the various bonding environments in carbon. 

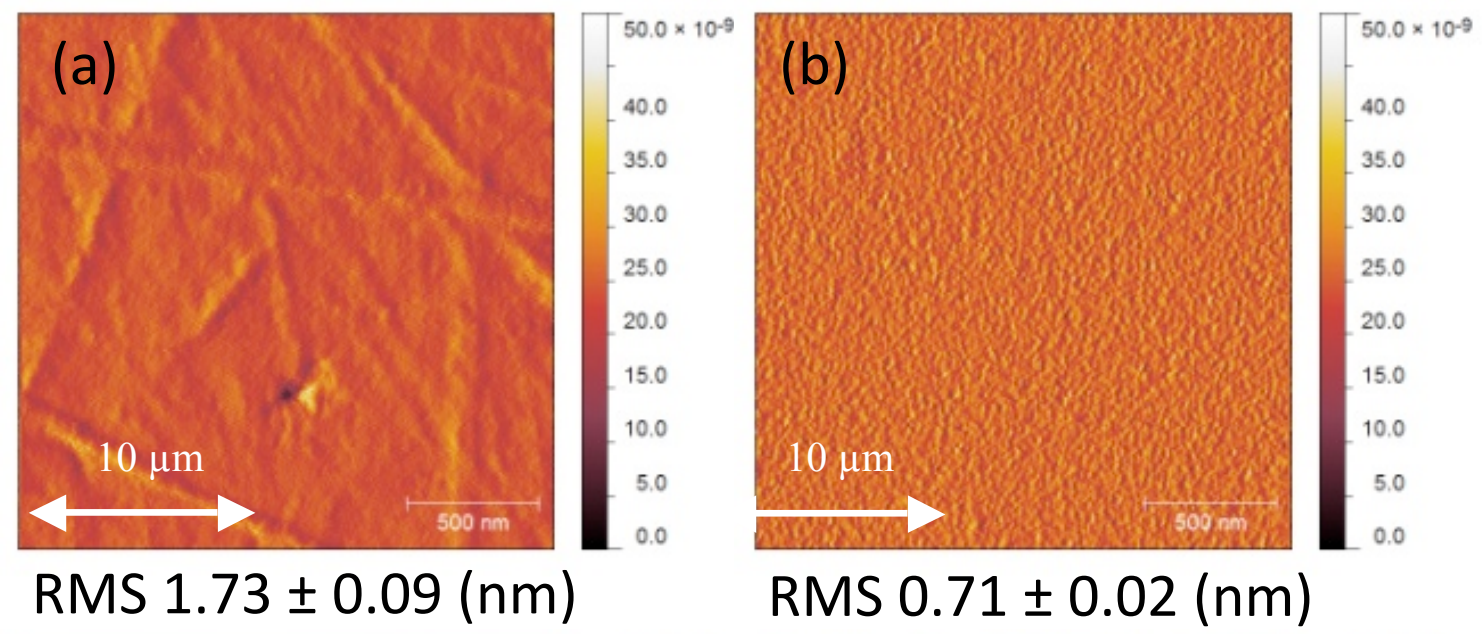

RMS $0.71 \pm 0.02(\mathrm{~nm})$
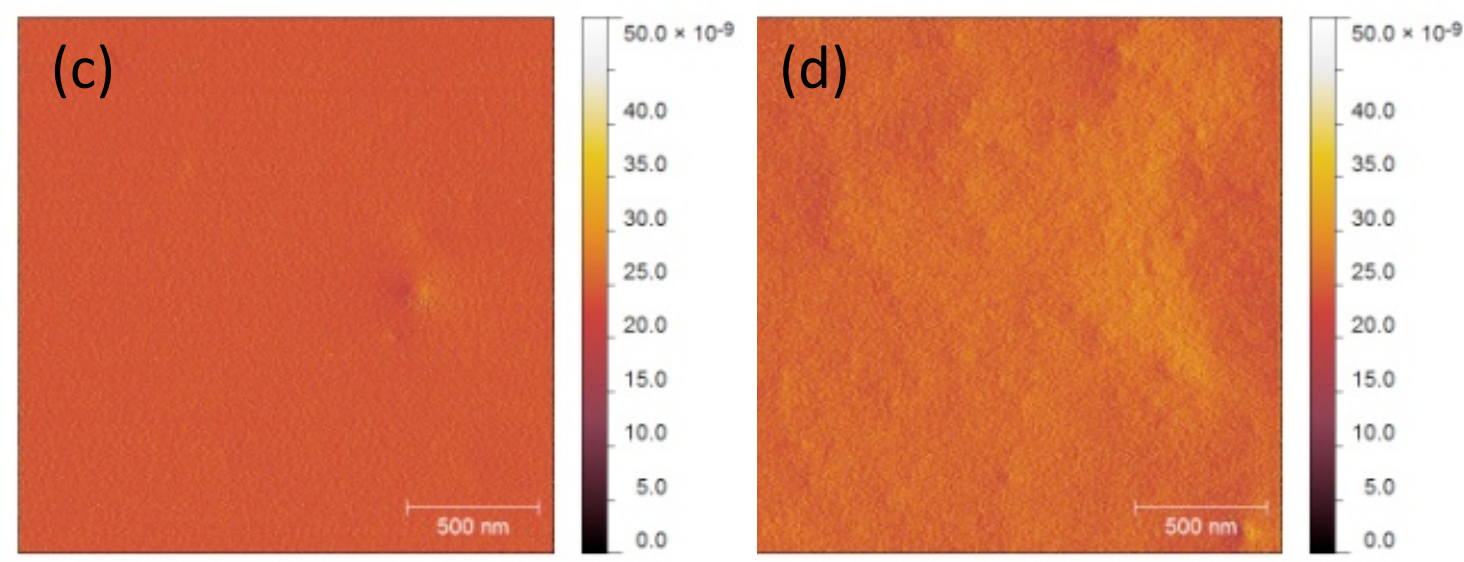

RMS $0.49 \pm 0.20(\mathrm{~nm})$

RMS $1.1 \pm 0.21(\mathrm{~nm})$

Figure 4. AFM image of plasma deposited (a) plain COP, (b) TEOS coating, (c) AA coating and (d) TEOS/AA coating acrylic acid and (b) TEOS \& AA coatings on COP 


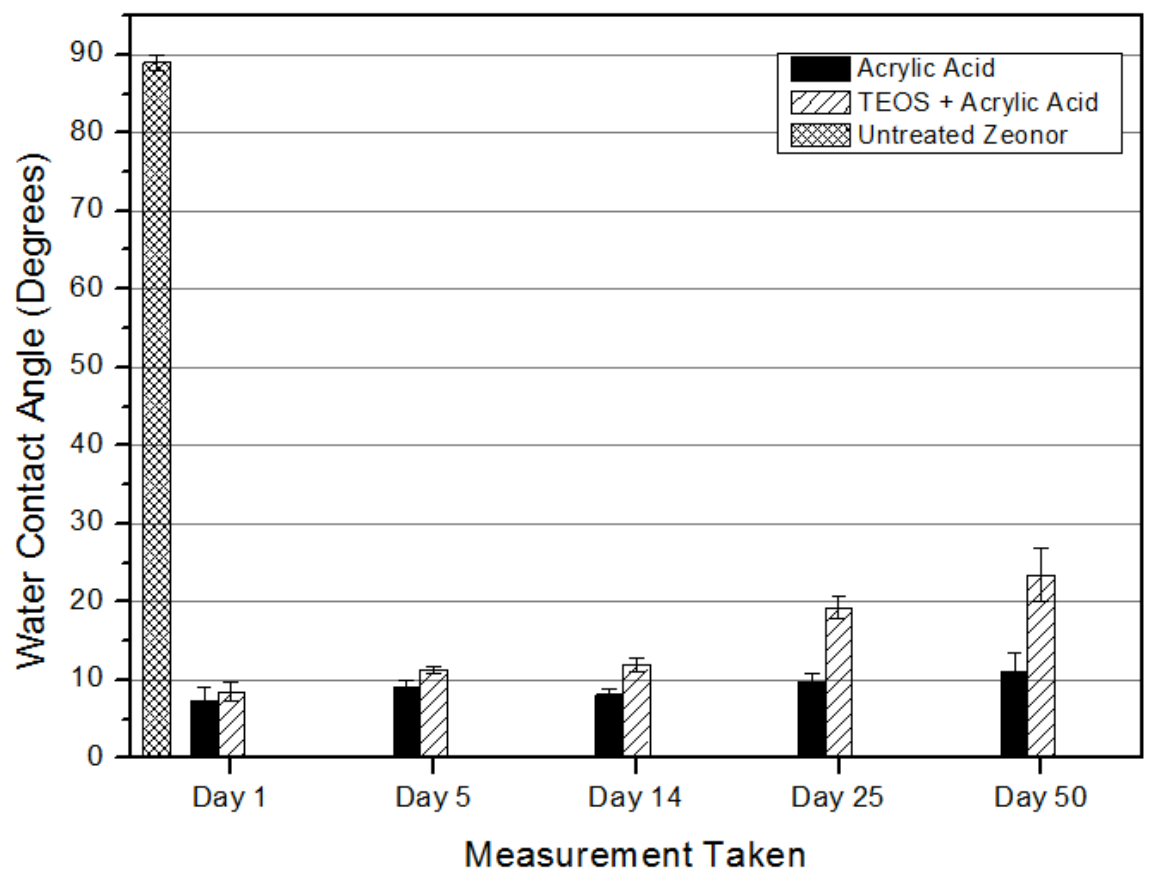

Figure 5 Variation of water contact angle with time (ageing) of the plasma deposited coatings on COP

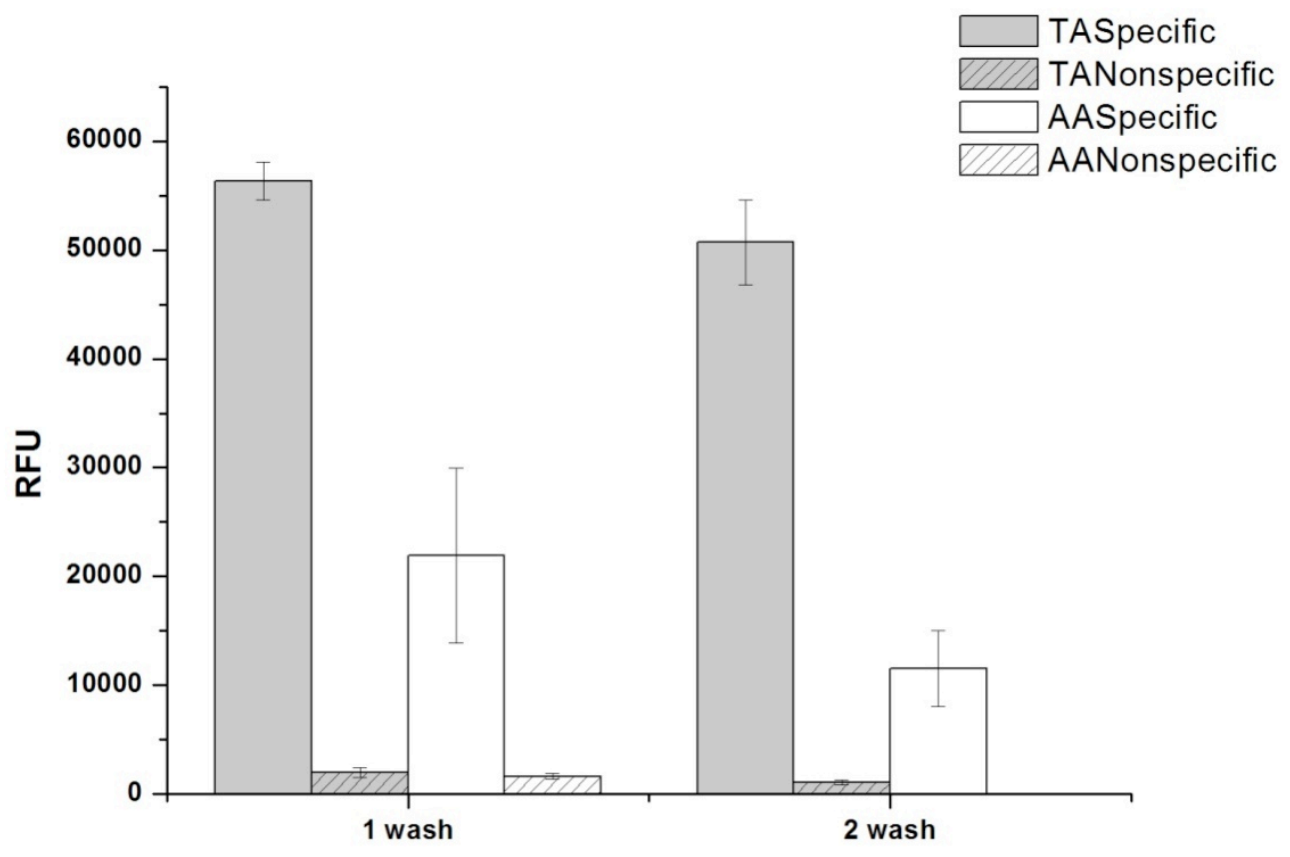


Figure 6 (a) Fluorescence intensity measurement of Cy5 labelled amino terminated DNA, with different wash steps, attached to plasma deposited coatings on COP with and without EDC NHS activation (b) signal to noise ratio
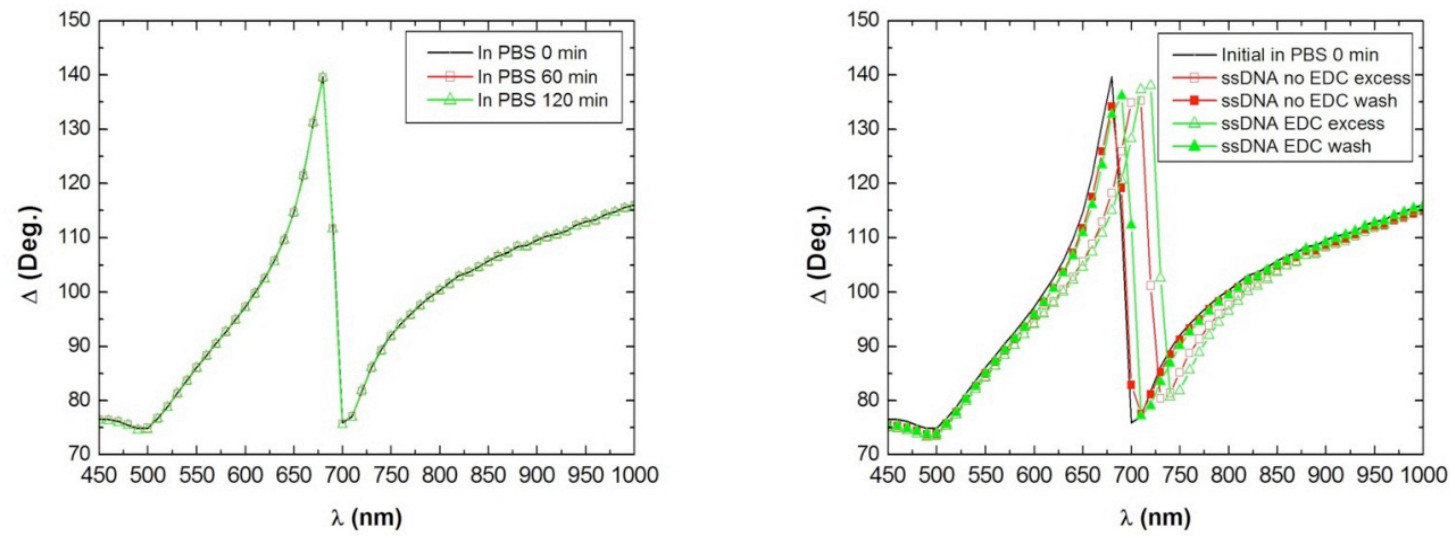

Figure 7. (a) $\Delta$ spectra measured on TEOS \& AA functionalized COP surface first filled with PBS and replaced with new PBS after $1 \mathrm{~h}$ and $2 \mathrm{~h}$. (b) $\Delta$ spectra measured on original TEOS \& AA functionalized COP surface first filled with PBS buffer (representative from well 2), then reacted with excess ssDNA $10 \times 10^{-6} \mathrm{M}$ without EDC (well 2) and with EDC (well 3), and after rinsing with PBS buffer.
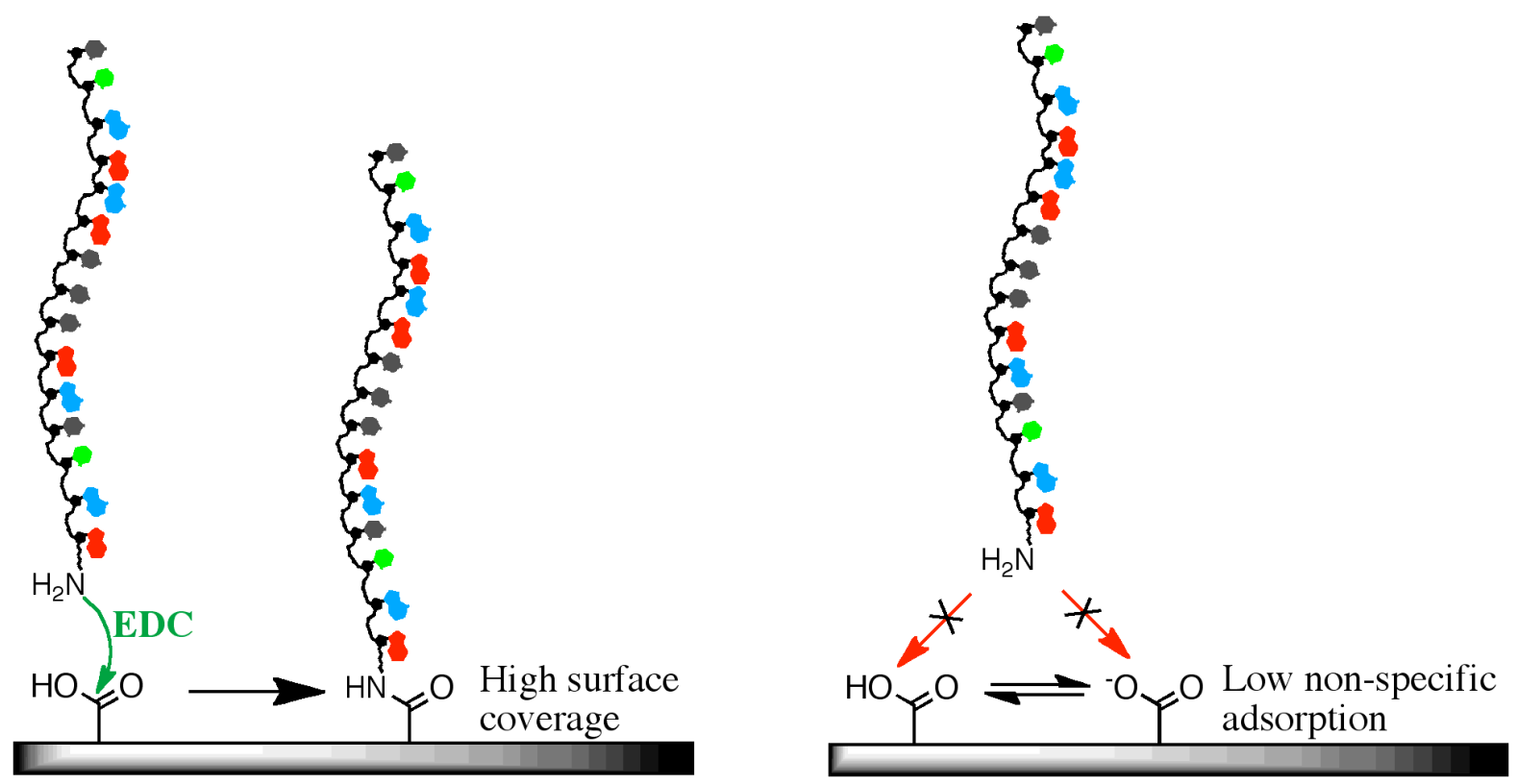
Abstract Figure. Left - A schematic illustration of specific immobilization of aminomodified DNA strand catalyzed by dehydrating agents such as EDC; and Right - low nonspecific adsorption of the DNA strand in the absence of activating reagents.

References:

Advincula ,M., Fan, X., Lemons, J., Advincula, R., Colloids Surf. B: Biointerfaces. 2005. 42, 29-43.

Alexander, M.R., Duc, T.M., J. Mater. Chem. 1998. 8, 937-943.

Azzam, R.M.A., Bashara, N.M., Ellipsometry and polarized light, North Holland, third printing, 1992.

Beaulieu, I., Geissler, M., Mauzeroll, J., Langmuir 2009. 25, 7169-7176.

Becker, H., Gartner, C., Anal. Bioanal. Chem. 2008. 390, 89-111.

Detomaso, L., Gristina, R., Senesi, G.S., d'Agostino, R., Favia, P., Biomaterials. 2005a. 26, 3831-3841.

Detomaso, L., Gristina, R., Senesi, G.S., d'Agostino, R., Favia, P., Surf. Coat. Technol. 2005b. 200, 1022-1025.

Diaz-Quijada, G.A., Peytavi, R., Nantel, A., Roy, E., Bergeron, M.G., Dumoulin, M.M., Veres, T., Lab Chip 2007. 7, 856-862.

Dudek, M.M., Gandhiraman, R.P., Volcke, C., Daniels, S., Killard, A.J., Plasma Process. Polym. 2009. 6, 620-630.

Gandhiraman, R.P., Volcke, C., Gubala, V., Doyle, C., Basabe-Desmonts, L., Dotzler, C., Toney, M.F., Iacono, M., Nooney, R.I., Daniels, S., James, B., Williams, D.E., J. Mat. Chem. 2010a. 20, 4116.

Gandhiraman, R.P., Gubala, V., Le Hoai, N.C., Volcke, C., Doyle, C., James, B., Daniels, S., Williams, D.E., Colloids. Surf., B 2010b. 79, 270-275. 
Gandhiraman, R.P., Karkari, S.K., Daniels, S.M., MacCraith, B.D., Surf. Coat. Tech. 2009c. 203, 3521- 3526 .

Gubala, V., Gandhiraman, R.P., Volcke, C., Doyle, C., Coyle, C., James, B., Daniels, S., Williams,D.E., Analyst. 2010. 135, 1375-1381.

Gupta, B., Plummera, C., Bissonb, I., Freyb, P., Hilborn, J., Biomaterials. 2001. 23, 863-871.

Hoai Le, N.C., Gubala, V., Gandhiraman, R.P., Coyle, C., Daniels, S., Williams, D.E., Anal. Bioanal. Chem. 2010. 398, 1927-1936

Hu, J., Wang, M., Weier, H.-U.G., Frantz, P., Kolbe, W., Ogletree, D.F., Salmeron M., Langmuir. 1996. 12 (7), 1697-1700.

Kasry, A., Borri, P., Davies,P.R., Harwood, A., Thomas, N., Lofas, S., Dale, T., Appl. Mater. Interfaces. 2009. 1 (8), 1793-1798.

Larsson, A., Ekblad, T., Andersson, O., Liedberg, B., Biomacromolecules. 2007. 8, 287-295. Liu, D., Bruckbauer, A., Abell, C., Balasubramanian, S., Kang, D.-J., Klenerman, D., Zhou, D., J. Am. Chem. Soc. 2009. 131 (34), 12211-12217.

Ma, K.S., Reza,F., Saaem, I., Tian, J., J. Mater. Chem. 2009. 19, 7914-7920.

Morra, M., Occhiello, E., Garbassi, F., Die Angewandte Makromol. CheM. 1991. 189, 125136.

Nabok, A., Tsargorodskaya, A., Davis, F., Higson,S.P.J., Biosens. Bioelectron., 2007. 23, $377-383$

Palumbo, F., Favia, P., Rinaldi, A., Vulpio, M. d'Agostino, R. Plasmas Polymers 1999. 4, $133-145$.

Pasternack, R.M., Amy,S.R., Chabal,Y.J., Langmuir. 2008. 24 (22), 12963-12971.

Poksinski., M., Arwin, H., Opt. Lett.32, 2007. 1308-1310.

Raj, J., Herzog, G., Manning, M., Volcke, C., MacCraith, B.D., Ballantyne,S., Thompson, M., Arrigan, D.W.M., Biosens. Bioelectron. 2009. 24, 2654-2658. 
Rechendorff, K., Hovgaard, M.B., Foss, M., Zhdanov, V.P., Besenbacher, F., Langmuir. 2006. $22,10885-10888$.

Roy, S., Chen, X.,Li, M.-H., Peng, Y., Anariba, F., Gao, Z., J. Am. Chem. Soc. 2006. 128 (6), 2067-2071.

Volcke, C., Gandhiraman, R.P., Gubala, V., Raj, J., Cummins, T., Fonder, G., Nooney, R.I., Mekhalif, Z., Herzog, G., Daniels, S., Biosens. Bioelectron. 2010. 25, 1875-1880. 